\title{
The First Case of Gastric Neuroendocrine Tumors Induced by a Proton Pump Inhibitor in von Hippel-Lindau Disease
}

\author{
Koichiro Kawaguchi ${ }^{1}$, Kazuo Yashima ${ }^{1}$, Yuichiro Ikebuchi ${ }^{1}$, Akira Yoshida ${ }^{1}$, \\ Satoshi Kuwamoto ${ }^{2}$ and Hajime Isomoto ${ }^{1}$
}

\begin{abstract}
:
Von Hippel-Lindau (VHL) disease is a rare inherited familial syndrome complicated with various neoplasms, including neuroendocrine tumors (NETs). We herein report the first case of multiple gastric NETs in a 45-year-old man with VHL. He had multiple gastric polyps, and several endoscopic resected lesions were diagnosed as NETs. The serum gastrin level was elevated because he was taking a proton pump inhibitor (PPI). We suspected that gastrin had played a role in the development of NETs, and the remaining polyps were followed up with discontinuation of the PPI. The NETs gradually reduced in size until they became hard to notice on endoscopy and have remained nearly invisible for over eight years.
\end{abstract}

Key words: von Hippel-Lindau disease (VHL), neuroendocrine tumor (NET), proton pump inhibitor (PPI), gastrin

(Intern Med Advance Publication)

(DOI: 10.2169/internalmedicine.8701-21)

\section{Introduction}

Von Hippel-Lindau (VHL) disease is an autosomaldominant familial disorder in which affected individuals are predisposed to develop a variety of tumors in multiple organ systems, with an incidence of 1 in 36,000 of all live births (1). It is caused by a germline mutation in the VHL tumor suppressor gene located on chromosome $3 \mathrm{p} 25$, which consists of 3 exons and encodes the tumor-suppressive VHL protein (p-VHL). pVHL plays a central role in the mammalian oxygen-sensing pathway, through ubiquitination of hypoxia-inducible factors (HIFs) (2). Dysregulation of this cascade leads to stabilization of HIFs and transcriptional activation of their target genes, which play a role in tumorigenesis and angiogenesis (3).

VHL-associated tumors include craniospinal and retinal hemangioblastomas, pancreatic tumors and cysts, renal cell carcinomas and cysts, pheochromocytomas, endolymphatic sac tumors, and broad ligament/epididymal cystadenomas (1). Neuroendocrine tumors (NETs), which were previously called carcinoids, have been rarely reported in VHL patients, and most of them are seen in the pancreas $(4,5)$. The association between VHL gene mutations and NETs had not been reported until recently. Pancreatic NETs are one of the tumors whose genome has recently been sequenced, and several mechanisms involved in VHL disease have thus been elucidated.

and several mechanisms involved in VHL disease have been elucidated (4).

NETs are rare tumors and can occur in various parts of the body, with the most common primary site being the gastrointestinal tract (6). Gastric NETs are well-differentiated neuroendocrine neoplasms arising from the non-peptidesecreting and thus non-functional generated endocrine cells of the gastric mucosa, which are usually derived from enterochromaffin-like (ECL) cells. Gastric NETs develop under three distinct circumstances, each with a different disease association, pathology, and prognosis (7).

We herein report a case of multiple gastric NETs in a patient with VHL.

\footnotetext{
${ }^{1}$ Division of Gastroenterology and Nephrology, Faculty of Medicine, Tottori University, Japan and ${ }^{2}$ Department of Pathology, Faculty of Medicine, Tottori University, Japan

Received: September 20, 2021; Accepted: December 7, 2021; Advance Publication by J-STAGE: February 8, 2022

Correspondence to Dr. Koichiro Kawaguchi, koichiro@tottori-u.ac.jp
} 
VHL gene Exon 1

Control
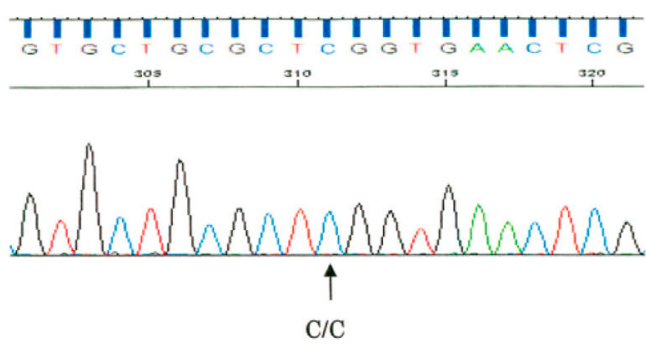

Patient

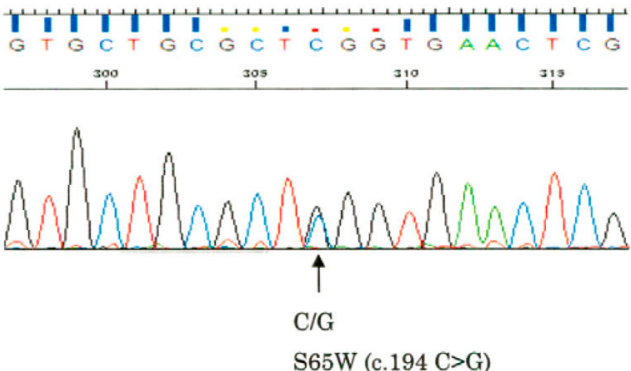

Figure 1. The germline mutation in the VHL tumor suppressor gene of the patient. The genetic test revealed a point mutation in VHL gene Exon1, S65W (c.194 C>G) in this patient, the same mutation as in his daughter.
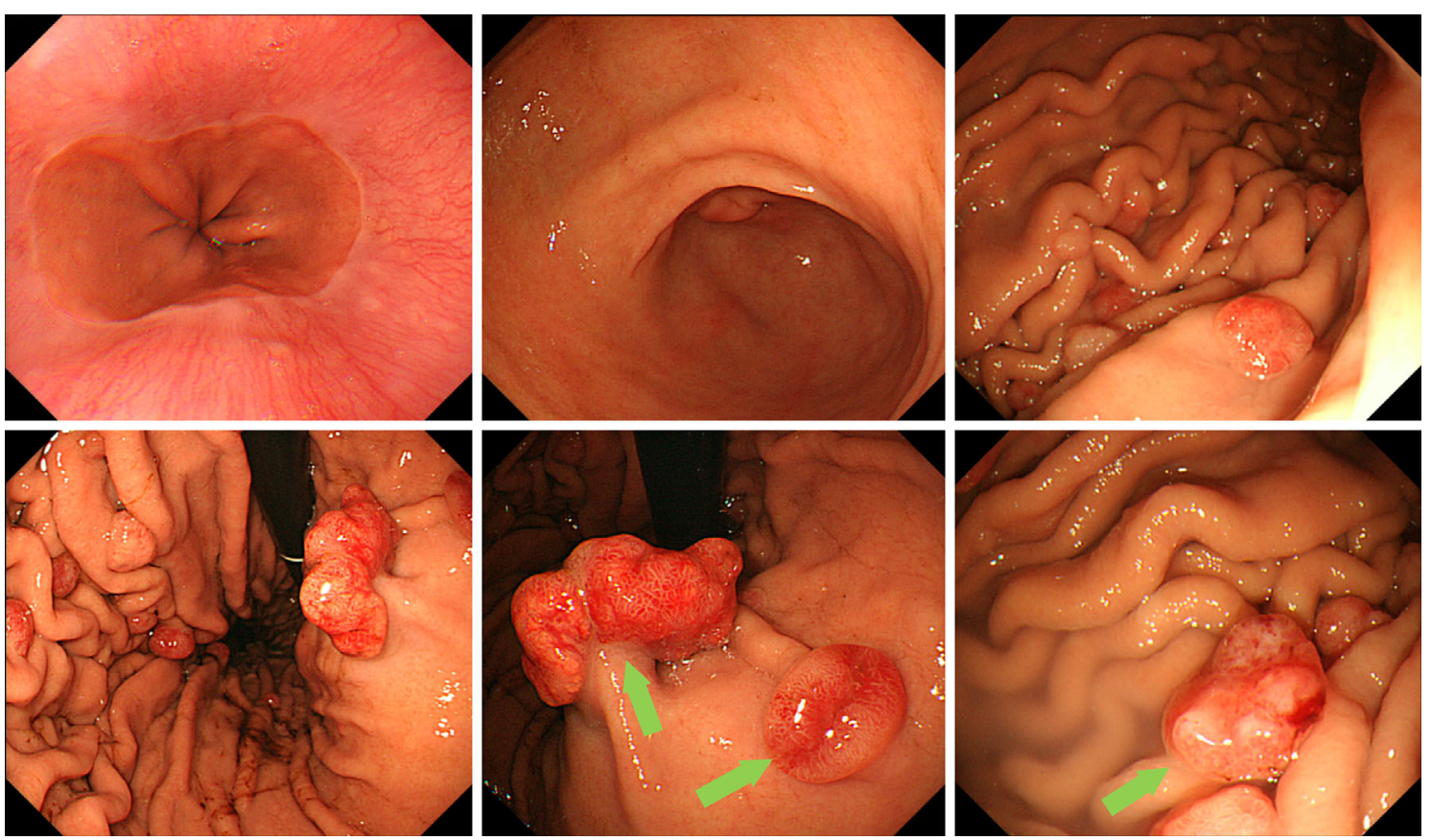

Figure 2. Endoscopic findings of gastric polyps before treatment. There is no atrophy in the stomach, and multiple polyps are seen on both the lesser and greater curvature of the body. The morphology of the polyps was very similar to hyperplastic polyps, i.e., they appeared to be erythematous (reddish), Isp-type epithelial tumors. In addition, three tumors (arrows) were subjected to EMR.

\section{Case Report}

\section{The Patient's History and Examination Findings}

A 45-year-old man had been diagnosed with VHL disease at 31 years old by genetic testing in the year 2000. He was on maintenance hemodialysis following total nephrectomy for bilateral renal cell carcinomas and had undergone several rounds of resection for multiple cerebellar hemangioblastomas. The family had a strong history of VHL; his father, brother, and daughter had suffered from VHL. Furthermore, a genetic test confirmed that the germline mutation in the VHL tumor suppressor gene was the same as VHL gene
Exon1, S65W (c.194 C>G), which was present in both this patient and his daughter (Fig. 1).

He was also on an antiplatelet agent (clopidogrel $75 \mathrm{mg}$, oral administration) for angina pectoris and had been taking a proton pump inhibitor (PPI; lansoprazole $15 \mathrm{mg}$ oral administration) since June 2006 to prevent gastro-duodenal ulcer. In April 2013, severe anemia was detected, with a hemoglobin level below $10 \mathrm{~g} / \mathrm{dL}$ that subsequently decreased to $7.7 \mathrm{~g} / \mathrm{dL}$. He therefore underwent a digestive tract checkup at our department. There were no significant findings on colonoscopy, but multiple polyps resembling hyperplastic polyps were seen in the body of the stomach on esophago-gastro-duodenoscopy (EGD) (Fig. 2). No atrophy was observed in the background gastric mucosa, which was 


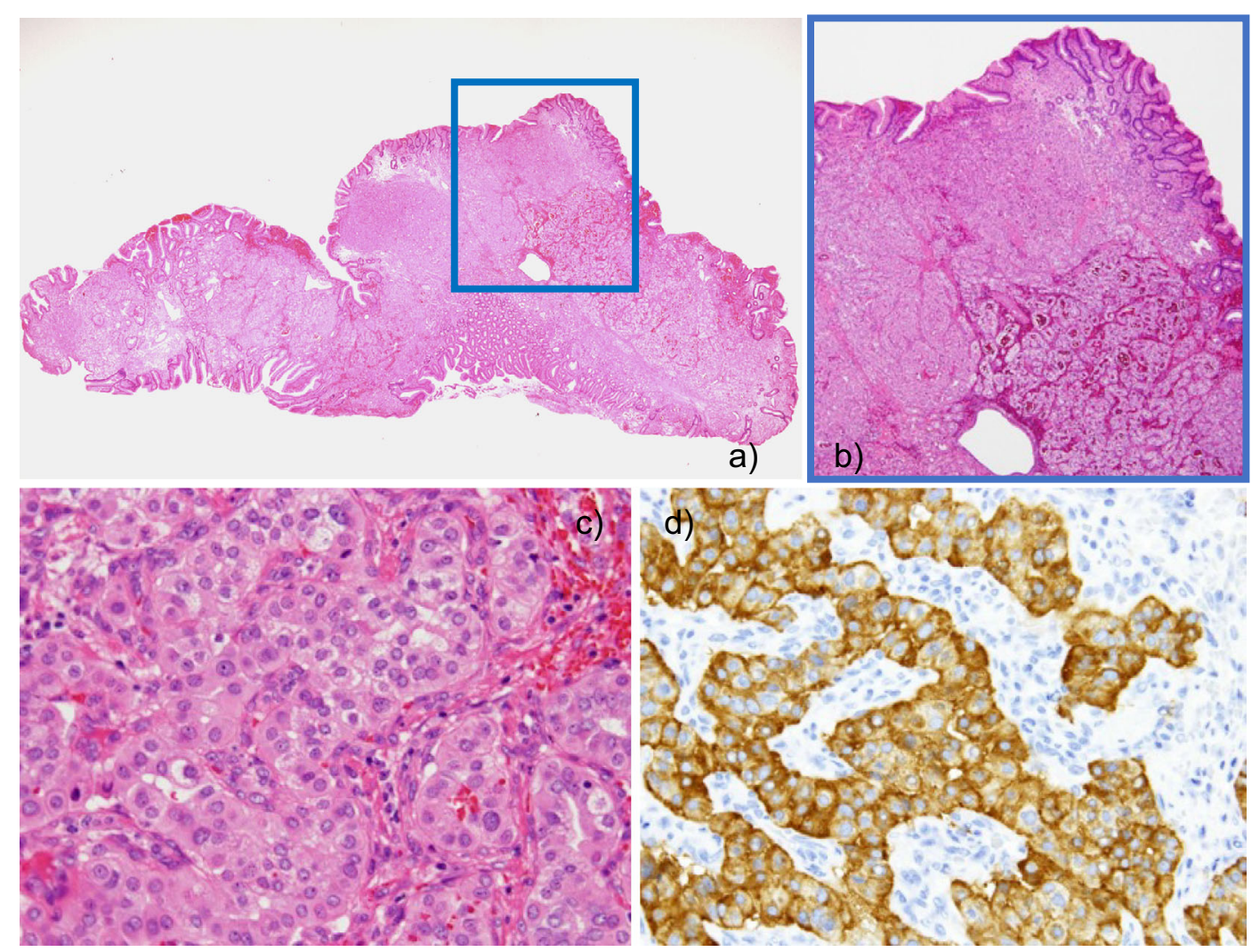

Figure 3. Pathological findings of the resected lesions. a) Loupe statue of the excised specimen. b) Hematoxylin and Eosin staining with a low-power field showed that the excised specimen was covered with normal mucosa of the fundic gland region on the surface layer. c) Tumor cells with a circular nucleus and abundant foamy or granular cytoplasm were observed inside. d) Immunohistochemical staining was positive for Synaptophysin.

C-0 according to the Kimura-Takemoto endoscopic classification (8). There was no mucosal swelling, diffuse redness, enlarged folds, or sticky mucus, which are considered indicative of a current infection with Helicobacter pylori according to the Kyoto classification of gastritis (9). Furthermore, no mucosal changes, such as cracked mucosa or cobblestone-like mucosa, which are associated with the long-term administration of PPIs, were observed $(10,11)$ (Fig. 2). Notably, EGD previously conducted in 2002 had shown no abnormalities, such as polyps or atrophy.

Blood investigations were negative for $H$. pylori infection (anti-H. pylori antibody $<3 \mathrm{U} / \mathrm{mL}$ ). Both anti-parietal cell antibody and anti-intrinsic factor antibody were negative, and pepsinogen levels were within the normal range when measured at a later date.

\section{Endoscopic Treatment and Pathological Findings}

We suspected these lesions to be hyperplastic polyps, and the administration of clopidogrel may have increased the bleeding from these polyps, thus resulting in the development of anemia.

Three large lesions (arrow in Fig. 2) were thus subjected to endoscopic mucosal resection. Histopathological and immunohistochemical examinations of the resected lesions were suggestive of NET.
The excised specimen was covered with normal mucosa of the fundic gland region on the surface layer, and tumor cells with a circular nucleus and abundant foamy or granular cytoplasm were observed inside. Partial tumor cell polymorphism was observed; however, there was no necrosis. A fissile image of 2/10 high-power fields was evaluated, and the maximum MIB-1 index was $4.4 \%$. Immunostaining was positive for the endocrine markers CD 56, Chromogranin A, and Synaptophysin, and gastrin was negative. These findings were equivalent to NET grade 2. The stump was negative at both the horizontal and vertical tumor margins (Fig. 3).

\section{Follow-up for the Remaining NETs of the Stomach}

Although the serum gastrin level was slightly elevated at $270 \mathrm{pg} / \mathrm{mL}$ (normal range $<200 \mathrm{pg} / \mathrm{mL}$ ), we suspected that gastrin might be associated with the progression of these NETs, as is seen in chronic atrophic gastritis type A. The remaining lesions were therefore followed up after discontinuation of the PPI, avoiding gastrectomy. The size of the tumors gradually decreased in line with the gastrin level (gastrin at 3 months later: $120 \mathrm{pg} / \mathrm{mL}$ ) until they met the normal range of the international criteria; eventually, most lesions were difficult to detect endoscopically (6 months later) (Fig. 4). The gastrin level was normalized, and the anemia was under control. 


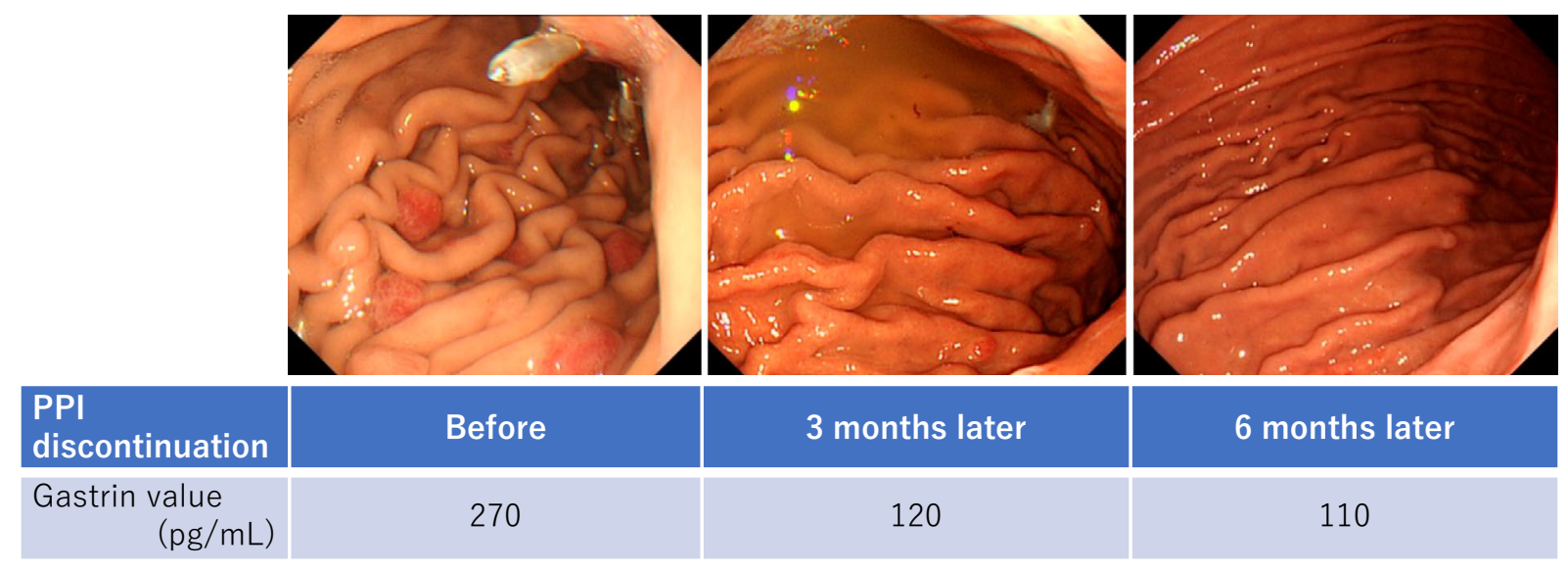

Figure 4. There were multiple NETs remaining in the stomach after EMR following PPI therapy; however, three months after the discontinuation of PPI, the lesions were significantly reduced in both size and number, along with normalization of gastrin levels, and after six months, most of them were not visible.
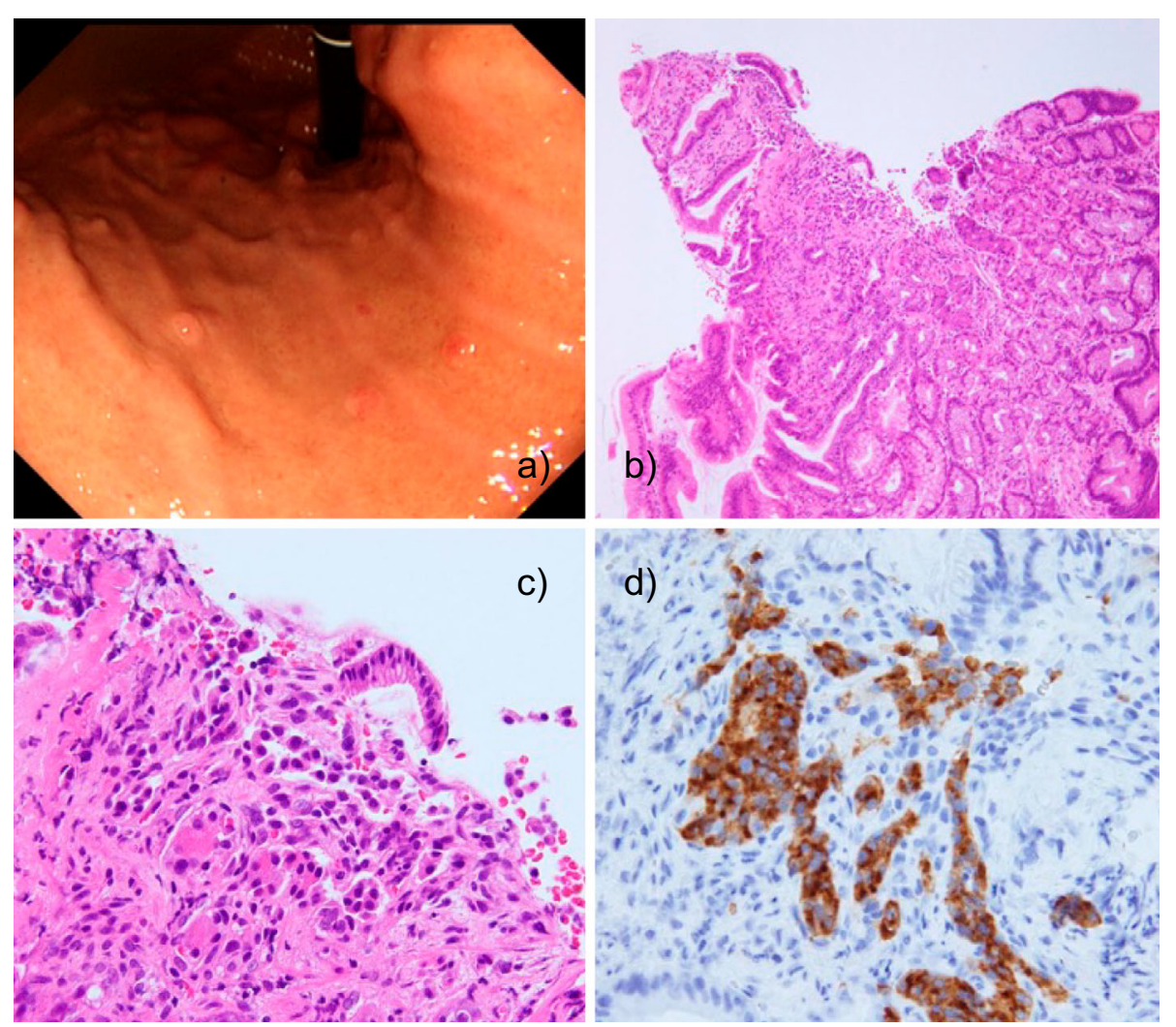

Figure 5. At one year after treatment, the reduction in tumor size was sustained (a). When a small lesion was biopsied, the surface layer was covered with normal mucosa with erosion (b), but a few small clusters of cuboidal cells with small round nuclei were observed in the deep part of the lamina propria (c). On immunostaining, the cells were positive for Synaptophysin (d).

While most lesions were not detectable endoscopically, tumor cells were detected on a biopsy of several remaining lesions, and these cells were diagnosed as NETs by immunostaining (Fig. 5). However, the tumors were very small $(<0.5 \mathrm{~mm})$ and were seen as small clusters of cells that were positive on immunostaining for Synaptophysin and Chromogranin A, and negative for gastrin.

The endoscopy interval was expanded from every three months for the first two years to every six months after two years, and every year after four years. Notably, the most recent EGD still showed no gastric atrophy (Kimura-Takemoto classification type C-0). At present, his gastric NETs have remained unchanged over eight years. Although they were still detectable as very small SMTs on his most recent endoscopy, biopsies did not show any NET cells.

We have been carefully following this patient with cessa- 
tion of acid suppression therapy and monitoring for recurrence or generation of the VHL tumors.

\section{Discussion}

NETs in other organs aside from the pancreas in patients with VHL are extremely rare (5), and there have been no reports of gastric NETs in patients of VHL. To our knowledge, this patient is the first with multiple gastric NETs associated with VHL. Furthermore, we suspect that the increase in gastrin associated with acid suppression therapy may have been involved in the development of these NETs. No gastric tumors were identified in the patient before PPI administration, whereas multiple NETs were found during preventive PPI therapy. In addition, the gastric NETs were almost undetectable on EGD after the discontinuation of the PPI.

Gastric NETs account for $<1 \%$ of gastric neoplasms (6). Classically, they can be separated into three distinct groups based on their clinical and histological characteristics: those associated with chronic atrophic gastritis type A (type I), those associated with the Zollinger-Ellison syndrome (type II), and sporadic gastric carcinoid tumors (type III). Type I and II NETs are multifocal and accompanied by hypergastrinemia. These NETs are almost invariably surrounded by areas of ECL cell hyperplasia, and these hyperplastic lesions can develop into carcinoid tumors $(6,7,12)$. We believe that the present case had an identical pathophysiology to these pathological conditions. We hypothesized that the gastrin levels would decline with the discontinuation of the PPI, causing the patient's gastric NETs to decrease in size and number or even disappear altogether. Experimentally, it is well known that in rats, carcinoids derived from ECL cells are generated by the administration of a large amount of a PPI. However, in healthy humans, although hyperplasia of ECL cells occurs, this is unlikely (13).

According to our literature search, five cases of non-VHL NET in patients taking PPIs have been reported (14-17). However, all of these cases had a single NET lesion, and the gastrin levels were either not high $(n=3)$ or not measured ( $=2$ ). One was a case of neuroendocrine carcinoma, which is considered to be unrelated to hypergastrinemia (17). It is difficult to confirm whether or not the NET in these cases was related to PPI administration. Nevertheless, in our case, we suspect that PPI was definitely associated with the occurrence of the NETs, as the tumors reduced in both size and number following the discontinuation of the PPI.

In this patient, NET cells were found on repeat biopsies, although the lesions were almost invisible on EGD. This is similar to a condition called endocrine cell micronest (ECM), which is frequently seen in chronic atrophic gastritis type A (18). Although there were no gross gastric polyps before PPI administration, whether the precursor lesions (like ECM seen in chronic atrophic gastritis type A) of NET developed due to long-term PPI administration or originally existed as a genetic factor associated with VHL is unclear.
At the very least, no increase in fundic gland polyps or changes in the background gastric mucosa (known as a 'cobblestone-like appearance'), which are observed with long-term administration of PPIs in healthy subjects without $H$. pylori infection, have been observed.

The level of circulating gastrin was not very high in this case; in fact, it was rather low compared to the level seen in patients taking a full-dose PPI. It is unlikely for ECL cells to be stimulated by such gastrin levels and then progress to NETs under normal circumstances. However, the lesions in our patient progressed to NETs despite receiving only a maintenance dose of lansoprazole, possibly because the patient had VHL. In VHL disease, the VHL protein forms a complex with several binding proteins controlling the aforementioned HIF cascade, which leads to tumorigenesis. Under normal circumstances, HIF can coordinate the cells' response to hypoxia. Through transcriptional regulation, HIF enhances the glucose uptake and increases the expression of angiogenesis, cellular growth, and mitogenic factors, including vascular endothelial growth factor (VEGF), plateletderived growth factor $\beta$ polypeptide (PDGF- $\beta$ ), erythropoietin, and transforming growth factor- $\alpha$ (TGF- $\alpha$ ). However, due to the abnormal or absent VHL protein function, HIF is overexpressed to induce the expression of these factors, thereby causing the tumor to develop and proliferate, as shown in the previous literature (1-3).

The presence of growth factors and their receptors in human gastric carcinoids and experimental models was investigated. Among them, we focused on TGF- $\alpha$ produced by both parietal and ECL cells. TGF- $\alpha$ regulates the inhibitory effect on histamine secretion and proliferative effect on ECL cells (19). The rodent species mastomys spontaneously develops ECLomas despite being normogastrinemic. Factors other than gastrin are thus required. During the hypergastrinemia-driven induction of mastomys ECLomas, increased ECL cell content of the mitogenic agent TGF- $\alpha$ and of its specific EGF receptor has been documented (19). The expression of TGF- $\alpha$ and EGF-receptors has also been demonstrated in human midgut carcinoids, suggesting that TGF- $\alpha$ participates in the autocrine modulation of carcinoid growth (20). In fact, immunostaining has proven that the resection specimen in this case was positive for EGF receptors. In contrast, TGF- $\alpha$ is a tumor growth factor that is increased in VHL, as mentioned above. Overexpression of TGF- $\alpha$ is seen in VHL-related tumors, especially renal cell carcinoma and cell lines. TGF- $\alpha$ has been demonstrated to support tumor cell growth through an autocrine loop (21). In this case, as in these experimental models, we hypothesized that gastrin-dependent TGF- $\alpha$ production occurred first, followed by ECL cell proliferation due to TGF- $\alpha$ autocrine stimulation.

Although TGF- $\alpha$ overexpression in "healthy" human ECL cells has not yet been documented (22), ECL cells might have developed into NETs in our patient with VHL.

In summary, our patient had been taking an oral PPI for about seven years until the lesions were detected, and during 
this period, the ECL cells received prolonged stimulation from gastrin above the normal limit. This case also proves that gastric NETs in patients with VHL can be reversed by eliminating the cause of ECL cell proliferation. In addition, VHL disease itself appears to be a genetic carcinogenic abnormality. We believe that the gastric NETs in this patient developed due to a combination of stimulation from gastrin and the involvement of the patient's genetic abnormality. It also indicates that physicians should be aware that gastric NETs might develop and progress in patients with special conditions, possibly under an environment of acid suppression.

\section{Informed Consent}

We obtained written informed consent from the patient before treatment and before the report submission.

The authors state that they have no Conflict of Interest (COI).

\section{Acknowledgement}

We would like to express our sincere gratitude to Dr. Shingo Ashida and Dr. Keiji Inoue, Department of Urology, Kochi Medical School, for reviewing the patient's DNA.

\section{References}

1. Lonser RR, Glenn GM, Walther M, et al. von Hippel-Lindau disease. Lancet 361: 2059-67, 2003.

2. Ohh M, Park CW, Ivan M, et al. Ubiquitination of hypoxiainducible factor requires direct binding to the beta-domain of the von Hippel-Lindau protein. Nat Cell Biol 2: 423-7, 2000.

3. Kim WY, Kaelin WG. Role of VHL gene mutation in human cancer. J Clin Oncol 22: 4991-5004, 2004.

4. Speisky D, Duces A, Bièche I, et al; GTE Group, Groupe d'Etude des Tumeurs Endocrines. Molecular profiling of pancreatic neuroendocrine tumors in sporadic and Von Hippel-Lindau patients. Clin Cancer Res 18: 2838-49, 2012.

5. Leung RS, Biswas SV, Duncan M, Rankin S. Imaging features of von Hippel-Lindau disease. Radiographics 28: 65-79, 2008.

6. Kulke MH, Mayer RJ. Carcinoid tumors.. N Engl J Med 340: 858-68, 1999

7. Rindi G, Luinetti O, Cornaggia M, Capella C, Solcia E. Three subtypes of gastric argyrophil carcinoid and the gastric neuroendocrine carcinoma: a clinicopathologic study. Gastroenterology 104 :
994-1006, 1993.

8. Kimura K, Takemoto T. An endoscopic recognition of the atrophic border and its significance in chronic gastritis. Endoscopy 3: 8797, 1969.

9. Kyoto Classification of Gastritis (in Japanese). Kato M, Inoue K, Murakami K, Kamada T, Haruma K, Eds. Nihon Medical Center, Tokyo, 2014.

10. Miyamoto S, Kato M, Tsuda M, et al. Gastric mucosal cracked and cobblestone-like changes by the use of proton pump inhibitors. Dig Endosc 29: 307-313, 2017.

11. Takahari K, Haruma K, Ohtani H, et al. Proton Pump Inhibitor Induction of Gastric Cobblestone-like Lesions in the Stomach. Intern Med 56: 2699-2703, 2017.

12. Williams GT. Endocrine tumors of the gastrointestinal tractselected topics. Histopathology 50: 30-41, 2007.

13. Lundell L, Vieth M, Gibson F, Nagy P, Kahrilas PJ. Systematic review: the effects of long-term proton pump inhibitor use on serum gastrin levels and gastric histology. Aliment Pharmacol Ther 42: 649-663, 2015.

14. Dawson R, Manson JM. Omeprazole in oesophageal reflux disease. Lancet 356: 1770-1771, 2000.

15. Haga Y, Nakatsura T, Shibata Y, et al. Human gastric carcinoid detected during long-term antiulcer therapy of $\mathrm{H} 2$ receptor antagonist and proton pump inhibitor. Dig Dis Sci 43: 253-257, 1998.

16. Jianu CS, Fossmark R, Viset $T$, et al. Gastric carcinoids after longterm use of a proton pump inhibitor. Aliment Pharmacol Ther 36: 644-649, 2012.

17. Jianu CS, Lange OJ, Viset $T$, et al. Gastric neuroendocrine carcinoma after long-term use of proton pump inhibitor. Scand J Gastroenterol 47: 64-7, 2012.

18. Itsuno $M$, Watanabe $H$, Iwafuchi $M$, et al. Multiple carcinoids and endocrine cell micronests in type A gastritis. Their morphology, histogenesis, and natural history. Cancer 63: 881-90, 1989.

19. Modlin IM, Tang LH. The gastric enterochromaffin-like cell: an enigmatic cellular link.. Gastroenterology 111: 783-810, 1996.

20. Nilsson $\mathrm{O}$, Wängberg $\mathrm{B}, \mathrm{McRae} A$, et al. Growth factors and carcinoid tumours. Acta Oncologica 32: 115-124, 1993.

21. Knebelmann B, Ananth S, Cohen HT, et al. Transforming growth factor alpha is a target for the von Hippel-Lindau tumor suppressor. Cancer Res 58: 226-31, 1998.

22. Bordi C, D'Adda T, Azzoni C, Ferraro G. Pathogenesis of ECL cell tumors in humans. Yale J Biol Med 71: 273-84, 1998.

The Internal Medicine is an Open Access journal distributed under the Creative Commons Attribution-NonCommercial-NoDerivatives 4.0 International License. To view the details of this license, please visit (https://creativecommons.org/licenses/ by-nc-nd/4.0/).

\section{(C) The Japanese Society of Internal Medicine Intern Med Advance Publication}

